\title{
Association between IGF-1 polymorphisms and risk of osteoporosis in Chinese population: a meta-analysis
}

\author{
Shu-tao Gao ${ }^{1 \dagger}$, Zheng-tao $\mathrm{Lv}^{2 \dagger}$, Chuan-kun Zhou ${ }^{3}, \mathrm{Chao} \mathrm{Mao}^{1}$ and Wei-bin Sheng ${ }^{1 *}$
}

\begin{abstract}
Background: Several studies looking into the association between insulin-like growth factor-1 (IGF-1) gene polymorphisms and osteoporosis predisposition have been conducted among Chinese population with conflicting outcomes. The present systematic review and meta-analysis was performed to appraise and synthesize the existing evidence, so as to provide a more precise and reliable association between polymorphisms in IGF-1 gene and osteoporosis.
\end{abstract}

Methods: Five electronic databases including PubMed, EMBASE, ISI Web of Science, CNKI and Wanfang were systematically searched for potential studies. Summary odds ratio (OR) and corresponding $95 \%$ confidence interval ( $95 \% \mathrm{Cl})$ were calculated to evaluate the association. The best-matching genetic model of inheritance was determined using a genetic-model free approach.

Results: Six case-control studies comprising 2068 osteoporosis patients and 2071 healthy controls were obtained for the meta-analysis. Dominant model was confirmed to be the best-matching genetic model (TT $+\mathrm{TC}$ versus CC). The overall data suggested that rs35767 polymorphism was significantly associated with osteoporosis vulnerability (OR 1.21, $95 \% \mathrm{Cl} 1.07,1.37 ; P=0.002$ ). When stratifying the participants and performing subgroup-analysis according to source of patients, the result suggested that rs35767 was significantly correlated to osteoporosis in post-menopausal women subgroup (OR 1.29, 95\% Cl 1.08, 1.54; $P=0.005)$, but the correlation was not established in the subgroup of both gender (OR 1.14, 95\% Cl 0.96, 1.35; $P=0.12)$.

Conclusion: Taken together, the findings of our current study suggested a significant association between rs35767 polymorphism and risk of osteoporosis in Chinese post-menopausal women.

Keywords: IGF-1, Single nucleotide polymorphism, Osteoporosis, Meta-analysis

\section{Background}

Osteoporosis is a common disorder predominantly characterized by low bone mineral density (BMD) and microarchitectural deterioration of bone, which increases the bone fragility and leads to fracture. With the prolongation of human lifespan, more and more elderly people are suffering from osteoporosis. Osteoporosis has become a worldwide public-health problem $[1,2]$.

\footnotetext{
* Correspondence: wbsheng@vip.sina.com

${ }^{\dagger}$ Equal contributors

${ }^{1}$ Department of Spine Surgery, The First Affiliated Hospital of Xinjiang Medical University, Xinjiang 830054, China

Full list of author information is available at the end of the article
}

Similar to other complex chronic disease in the elderly, the precise etiopathogenesis underlying osteoporosis is also multifactorial. Risk factors such as genetics, age, physical activity, diet, the use of glucocorticoids and so forth have to be taken into account [3]. Among them, genetic factors are found to play a pivotal role in the occurrence of osteoporosis and have received highly attention [4]. Large-scale twin studies observed that the heritability of bone loss among postmenopausal women was up to $56 \%$ $[5,6]$. Many genes with innumerous gene polymorphisms have been identified to be covert risk alleles for osteoporosis vulnerability. Up to now, a series of candidate genes like vitamin D receptor $[7,8]$, estrogen receptors $[9,10]$, 
osteoprotegerin $[11,12]$, have been widely reported to be associated with osteoporosis.

Insulin-like growth factor-1 (IGF-1) is a single-chain polypeptide encoded by IGF-1 gene. IGF-1 is an important anabolic hormone and has the ability to regulate bone homeostasis and maintain skeletal architecture throughout adult life [13]. Literature reported that decreased serum concentration of IGF-1 strongly contribute to the occurrence of osteoporotic fractures in postmenopausal women [14]. Considering the vitally functional significance of the IGF-1, variations in the IGF-1 gene are possible susceptibility candidate loci for osteoporosis. In fact, Lee et al. had initially reported the relationship between IGF-1 gene polymorphisms and the BMD among postmenopausal women in 2002 [15]. Soon afterwards, a large sample studies led by Rivadeneira et al. [16] suggested that the microsatellite repeat polymorphism in the promoter of IGF-1 was linked to the BMD level and bone loss in postmenopausal women.

The single nucleotide polymorphisms (SNPs) located in IGF-1 gene also had been identified to be significantly associated with osteoporosis by $\mathrm{Li}$ and coworkers [17]. Subsequent replication studies tried to validate the association, but yielded conflicting results. Compared to a single gene-disease association study, a meta-analysis can strengthen the statistical power by combining individual study. A previous meta-analysis including four observational studies by Chen et al. [18] has been carried out. Considering the emergence of novel evidence on the association of IGF-1 polymorphisms and risk of osteoporosis, we set out to perform the present systematic review and meta-analysis to obtain a more credible association between IGF-1 polymorphisms and osteoporosis predisposition.

\section{Methods}

This systematic review and meta-analysis conformed to the Preferred Reporting Items for Systematic Review and Meta-Analyses (PRISMA) guidelines [19].

\section{Literature search strategy}

Five electronic databases including PubMed, EMBASE, ISI Web of Science, National Knowledge Infrastructure (CNKI), and Wanfang were methodically searched to identify genetic association studies involving the polymorphisms in IGF-1 gene published before November, 2017. For English databases, the search string was set using the combinations of Medical Subject Headings $(\mathrm{MeSH})$ and free words as below: (Single Nucleotide Polymorphism or polymorphism or SNP or SNPs or "Polymorphism, Single Nucleotide"[Mesh]) and ("Insulin-Like Growth Factor I"[Mesh] or Somatomedin C or insulin-like growth factor 1 or IGF-1) and ("osteoporosis" [Mesh] or osteoporo" or bone loss or bone or fracture or "Fractures, Bone"[Mesh] or bone density or "Bone Density"[Mesh] or bone mineral density). For Chinese databanks, we adopt the following key words: "IGF-1|"| and |"duo tai xing" and "gu zhi shu song". To prevent incomplete retrieval, the reference lists of related reviews and primary articles were manually searched to identify all potential studies. Two investigators (SG and ZL) accomplished the retrieving task independently.

\section{Inclusion and exclusion criteria}

Studies included in our meta-analysis had to satisfy all of the following criteria: (1) osteoporosis diagnosed on the basis of radiological examination; (2) published studies aimed to assess the correlation between IGF-1 gene polymorphisms and osteoporosis; (3) case-control designed studies; (4) sufficient data provided for the calculation of the crude odds ratios (ORs) and 95\% confidence intervals (95\% CIs). Correspondingly, studies were excluded from our analysis for the following reasons: (1) case report, comments, reviews, or animal studies; (2) family-based studies; (3) data overlapping with previous article; (4) studies with unavailable alleles as well as genotype frequencies. For any discrepancy during this process, a consensus was achieved after discussion.

\section{Methodological quality assessment}

The methodological quality of included studies was evaluated separately by two reviewers (SG and ZL) under the Newcastle-Ottawa Scale (NOS) for observational studies [20]. A 'star system' was applied to judge individual study on three broad perspectives: the selection of the case and control groups; the comparability of the case and control groups, and the ascertainment of either the exposure or outcome of interest for case-control studies (http://www. ohri.ca/programs/clinical_epidemiology/oxford.asp). Disagreements between two investigators were settled by discussion until consensus was reached.

\section{Data extraction}

In compliance with the predetermined selection criteria, the following information was meticulously extracted by two reviewers (SG and ZL) from all qualified articles independently: (1) surname of the first author; (2) year of publication; (3) country where the study was conducted; (4) ethnicity of enrolled subjects; (5) numbers of case and control participants; (6) gender of the enrolled patients; (7) diagnostic criteria of osteoporosis; (8) genotypes distribution of case and control participants; (9) HardyWeinberg Equilibrium (HWE) test results of control subjects. In case of any discrepancy during this process, two authors re-inspected the article together and reached an agreement by mutual discussion. 


\section{Statistical analysis}

HWE for the control participants of each included study was evaluated by Chi-square test to assess goodness of fit. The strength of association between polymorphism in IGF-1 gene and risk of osteoporosis was presented as crude ORs accompanied by the corresponding 95\% CI. To prevent an inflated false positive error rate, we did not conduct any assumption about the genetic model of inherence beforehand. The most appropriate genetic model was determined by a model-free approach [21]. If A variant was the gene of interest that could possibly lead to an increased or decreased risk of osteoporosis. OR1, OR2 and OR3 were calculated for genotypes AA versus aa, Aa versus aa, AA versus Aa for each polymorphism to capture the magnitude of genetic effect and to decipher the most plausible genetic model. Then the most reasonable genetic model of inherence was ascertained according to the associations between the three pairwise comparisons as follow:

(1) Recessive model: if OR $1=\mathrm{OR} 3 \neq 1$ and OR2 $=1$;

(2) Dominant model: if OR $1=\mathrm{OR} 2 \neq 1$ and OR3 $=1$;

(3) Complete over-dominant model: if OR1 $=1$, OR2 = $1 / \mathrm{OR} 3 \neq 1$;

(4) Co-dominant model: if OR1 $>$ OR2 $>1$ and OR1 > OR $3>1$, or $\mathrm{OR} 1<\mathrm{OR} 2<1$ and $\mathrm{OR} 1<\mathrm{OR} 3<1$.

After the underlying genetic model was verified, the counts of genotypes were collapsed into two categories to obtain the merged results. To evaluate the between-study heterogeneity, Q-statistical test and $\mathrm{I}^{2}$ test were employed. The randomeffect model and fixed-effect model were utilized for data combination in the presence $\left(P<0.1, \mathrm{I}^{2}>50 \%\right)$ or absence of heterogeneity $\left(P>0.1, \mathrm{I}^{2}<50 \%\right.$ indicates an acceptable heterogeneity) respectively [22, 23]. In the event of any statistically significant heterogeneity across studies, subgroupanalysis by source of the patients (both gender subjects or only post-menopausal women) were performed to look for the potential source of heterogeneity. The Leave-one-out sensitivity analysis was conducted by removing individual study one by one and reassessing the resulting effect on the estimate of overall effect. Egger's regression test and Begg's rank correlation test were exploited to estimate the publication bias (Stata version 12.0, Stata Corp LP, USA) [24]. In case that the number of included studies was smaller than ten, Trim and Fill method was also used to evaluate potential publication bias. Forest plots and funnel plot were originated from RevMan 5.3 software (Copenhagen: The Nordic Cochrane Centre, The Cochrane Collaboration, 2014).

\section{Results}

Literature search

Five online databases were hunted for potentially relevant studies and the initial literature. Combining the search results, a total of 228 records were yielded comprising 85 from PubMed, 22 from EMBASE, 112 from ISI Web of Science, 5 from CNKI and 4 from Wanfang, and no additional records were gained by the manual retrieval. After the first scanning, 58 duplicated records were rejected. Of the 170 potentially relevant remainders, a further $163 \mathrm{ob-}$ viously improper articles were erased after reading the titles and abstracts. Among the remaining 7 records for full-text assessment, 1 unrelated was eliminated according to the predetermined inclusion and exclusion criteria. Ultimately, 6 articles [17, 25-29] were incorporated into the systematic review and meta-analysis. The literature selection process was presented in Fig. 1.

\section{Main characteristics}

The main characteristics of included studies were shown in Table 1. Six case-control studies containing 2068 osteoporosis patients and 2071 healthy controls were identified and included in our meta-analysis. All the included studies were implemented across Chinese population, the sample-size ranged from 436 to 936 . Of the six studies, five $[17,25-27,29]$ referred to rs35767, rs2288377, and rs5742612. While Wei et al.'s study [28] focused on rs35767 and rs972936. Three studies [25, 27, 29] reported both male and female participants, yet the remaining three studies $[17,26,28]$ focused on the association in only post-menopausal women subjects. The genotype frequencies of both case and control groups were summarized in Table 1. Six studies all conformed to the HWE in terms of rs35767. However, the majority of the studies did not comply with HWE when it came to rs2288377 and rs5742612, thus we did not perform meta-analyses for these two loci in the following section. On the basis of the NOS, all the six studies obtained an average of 6.3 stars for methodological quality assessment (Table 2).

\section{Meta-analyses and subgroup-analyses}

Previous to pooling data of all the included studies, we carried out testable hypotheses to look for the bestmatching genetic model of inherence. The estimated OR1 (TT/CC: $1.45,95 \%$ CI 1.01, 2.07; $P=0.04$ ) and OR2 (TC/CC: $1.15,95 \%$ CI 1.01, 1.31; $P=0.03$ ) were both statistically significant whereas the estimated OR3 (TT/TC: $1.27,95 \%$ CI $0.95,1.69 ; P=0.11$ ) was insignificant, indicating that the dominant model was the most reasonable genetic model for meta-analysis. When using the dominant model, the counts of genotypes of TT and TC groups were combined and compared with CC groups. Since no between-study heterogeneity across the included studies was detected $\left(P=0.42, \mathrm{I}^{2}=0\right)$, the fixed-effect model was adopted for statistical analysis. The summarized OR suggested that there existed a statistically significant association between rs35767 in IGF-1 gene and the osteoporosis 


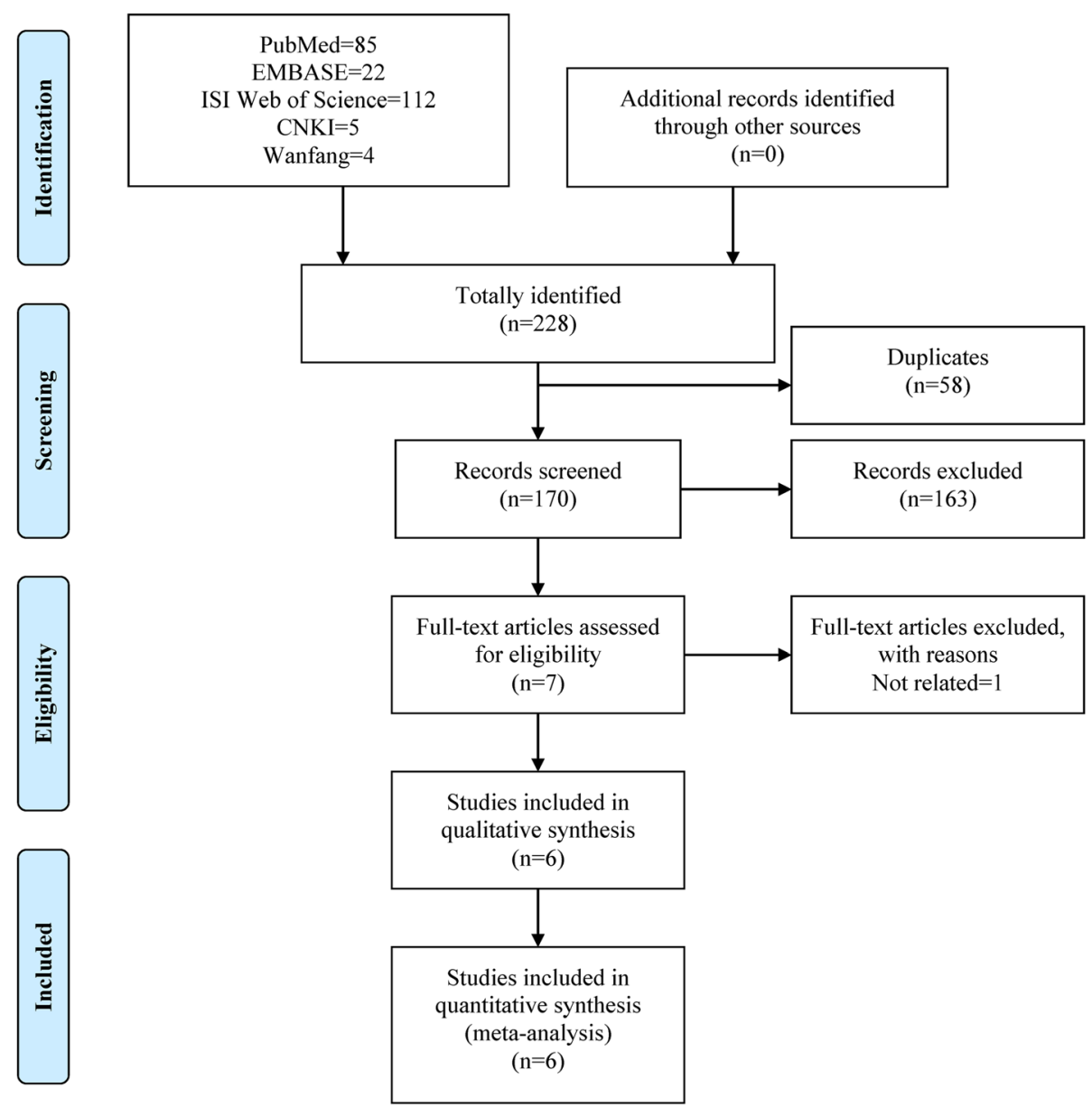

Fig. 1 Flow chart of literature search and screen

predisposition (OR 1.21, 95\% CI 1.07, 1.37; $P=0.002$ ) (Fig. 2).

Even though no heterogeneity was observed, we performed subgroup-analysis according to source of the patients to evaluate the association between rs35767 and osteoporosis susceptibility in both subgroups (Fig. 3). The results suggested that rs35767 was significantly correlated to the risk of osteoporosis in post-menopausal subgroup (OR 1.29, 95\% CI 1.08, 1.54; $P=0.005$ ), but the association was not established in the both gender subgroup (OR 1.14, 95\% CI 0.96, 1.35; $P=0.12$ ).

\section{Sensitivity analysis and publication bias}

The sensitivity analysis was performed by sequential excluding each of the eligible studies. While the corresponding pooled OR appeared to be not significantly affected, indicating a robust and stable result (Additional file 1: Figure S1). The funnel plot was visually symmetrical (Fig. 4), the Egger's test $(t=-0.64, P=0.555)$ and Begg's test $(\mathrm{z}=0.00, P=1.000)$ also suggested no statistically significant publication bias. Trim and Fill method showed that the association between rs35767 polymorphism and risk of osteoporosis was stable (number to trim $=0$, data were unchanged).

\section{Discussion}

With the general aging of societies, osteoporosis is growing into a serious health threat and has given rise to significant physical, psychosocial, and financial consequences to the sufferers. Given genetic factors play a leading role in the histogenesis and development of osteoporosis, identification of polymorphisms in potential pathogenicity genes enable us to predict the disease and take preventive measures, as well as determine potential targets for specific pharmacologic treatment. Association studies in human have defined a range of polymorphisms in several genes which regulate BMD and osteoporosis [30]. Among them, IGF-1 is one of the most commonly studied candidate genes due to its critical role in the regulation of bone turnover and homeostasis.

IGF-I is a ubiquitous hormone that expressed in virtually every tissue, but the circulating IGF-1 is mainly 
Table 1 Main characteristics of included studies

\begin{tabular}{|c|c|c|c|c|c|c|c|c|c|c|c|c|}
\hline \multirow{2}{*}{$\frac{\text { Study }}{\text { rs35767 }}$} & \multirow[t]{2}{*}{ Country } & \multirow[t]{2}{*}{ Ethnicity } & \multirow[t]{2}{*}{ Sample size } & \multirow[t]{2}{*}{ Source of patients } & \multirow[t]{2}{*}{ Diagnostic criteria } & \multicolumn{3}{|l|}{ Case } & \multicolumn{3}{|c|}{ Control } & \multirow[t]{2}{*}{ HWE } \\
\hline & & & & & & CC & $\mathrm{CT}$ & $\pi$ & CC & $C T$ & $\pi$ & \\
\hline Fan, 2017 & China & Chinese & $346 / 346$ & Both gender & WHO & 154 & 163 & 29 & 152 & 157 & 37 & 0.932 \\
\hline Li, 2014 & China & Chinese & $216 / 220$ & PMOP women & WHO & 95 & 94 & 27 & 114 & 89 & 17 & 0.998 \\
\hline Li, 2015 & China & Chinese & $485 / 485$ & PMOP women & WHO & 202 & 210 & 74 & 238 & 201 & 46 & 0.932 \\
\hline Liu, 2017 & China & Chinese & $320 / 320$ & Both gender & WHO & 152 & 136 & 32 & 156 & 131 & 33 & 0.781 \\
\hline Wei, 2015 & China & Chinese & $272 / 272$ & PMOP women & WHO & 124 & 118 & 30 & 132 & 116 & 24 & 0.979 \\
\hline Zhang, 2015 & China & Chinese & $428 / 428$ & Both gender & WHO & 182 & 193 & 53 & 216 & 186 & 26 & 0.233 \\
\hline rs2288377 & & & & & & $\mathrm{AA}$ & AT & $\pi$ & $\mathrm{AA}$ & AT & $\pi$ & \\
\hline Fan, 2017 & China & Chinese & $346 / 346$ & Both gender & WHO & 118 & 171 & 57 & 164 & 160 & 22 & 0.113 \\
\hline Li, 2014 & China & Chinese & $216 / 220$ & PMOP women & WHO & 182 & 21 & 13 & 189 & 21 & 10 & $<0.001$ \\
\hline Li, 2015 & China & Chinese & $485 / 485$ & PMOP women & WHO & 396 & 52 & 36 & 413 & 45 & 27 & $<0.001$ \\
\hline Liu, 2017 & China & Chinese & $320 / 320$ & Both gender & WHO & 125 & 145 & 50 & 157 & 138 & 25 & 0.781 \\
\hline Zhang, 2015 & China & Chinese & $428 / 428$ & Both gender & WHO & 349 & 44 & 35 & 365 & 40 & 23 & $<0.001$ \\
\hline \multicolumn{13}{|l|}{ rs5742612 } \\
\hline Fan, 2017 & China & Chinese & $346 / 346$ & Both gender & WHO & 298 & 30 & 18 & 301 & 35 & 10 & $<0.001$ \\
\hline Li, 2014 & China & Chinese & $216 / 220$ & PMOP women & WHO & 178 & 21 & 17 & 183 & 21 & 16 & $<0.001$ \\
\hline Li, 2015 & China & Chinese & $485 / 485$ & PMOP women & WHO & 389 & 55 & 41 & 400 & 50 & 35 & $<0.001$ \\
\hline Liu, 2017 & China & Chinese & $320 / 320$ & Both gender & WHO & 264 & 33 & 23 & 245 & 69 & 6 & 0.907 \\
\hline Zhang, 2015 & China & Chinese & $428 / 428$ & Both gender & WHO & 336 & 48 & 44 & 346 & 42 & 41 & $<0.001$ \\
\hline
\end{tabular}

PMOP: post-menopausal; WHO: World Health Organization criteria; HWE: Hardy-Weinberg Equilibrium

Statistically significant findings appeared in bold

produced and secreted in the liver tissue via the regulation of growth hormone [31].The mature IGF-1 polypeptide is encoded by exons 3 and 4 of IGF- 1 gene, which comprises six exons [13]. Both the systemic and local skeletal IGF-1 act as a central growth factor in the regulation of body formation and the maintenance of bone mass by enhancing bone matrix formation as well as inhibiting bone degradation $[13,32]$. It was estimated that more than $40 \%$ of basal bone cell proliferation could be blocked by inhibiting the actions of IGF-1 produced endogenously by bone cells in vitro study [33]. During the individual development and maturity, IGF-1 plays a key role in regulating longitudinal skeletal growth. Apart from this effect, IGF-1is much better known as an anabolic hormone which has the ability to determine bone modeling and remodeling throughout life. The anabolic effect of IGF-1 was mainly exerted on cortical and trabecular bone, and it is also of great importance for the achievement of the peak bone mass, which is a critical contributing factor for future risk of osteoporosis [34]. IGF-1 levels decrease with advancing age, Liu et al. [35] found the serum IGF-1 level started to decline at age 30, nearly 20 years earlier than the significant loss of BMD, thus making IGF-1 as a predictor for early bone loss. A large body of literature have also reported that IGF-1 and several IGF-binding proteins (IGFBPs), like IGFBP-1, 3, 4, and 5, were positively

Table 2 Methodological quality of included studies

\begin{tabular}{|c|c|c|c|c|c|c|}
\hline Item/Study & Fan, 2017 & $L i, 2014$ & Li, 2015 & Liu, 2017 & Wei, 2015 & O'Connell, 2014 \\
\hline Adequate definition of cases & * & * & * & * & * & * \\
\hline Representativeness of cases & - & - & - & - & * & - \\
\hline Selection of control subjects & * & - & - & - & - & - \\
\hline Definition of control subjects & * & * & * & * & * & * \\
\hline Control for important factor or additional factor & * & * & $* *$ & - & $* *$ & * \\
\hline Exposure assessment & * & * & * & * & * & * \\
\hline Same method of ascertainment for all subjects & * & * & * & * & * & * \\
\hline Non-response rate & * & * & * & * & * & * \\
\hline
\end{tabular}

A study could be awarded a maximum of one star for each item except for the item "Control for important factor or additional factor"

The definition/explanation of each column of the Newcastle-Ottawa Scale is available from http://www.ohri.ca/programs/clinical_epidemiology/oxford.asp 


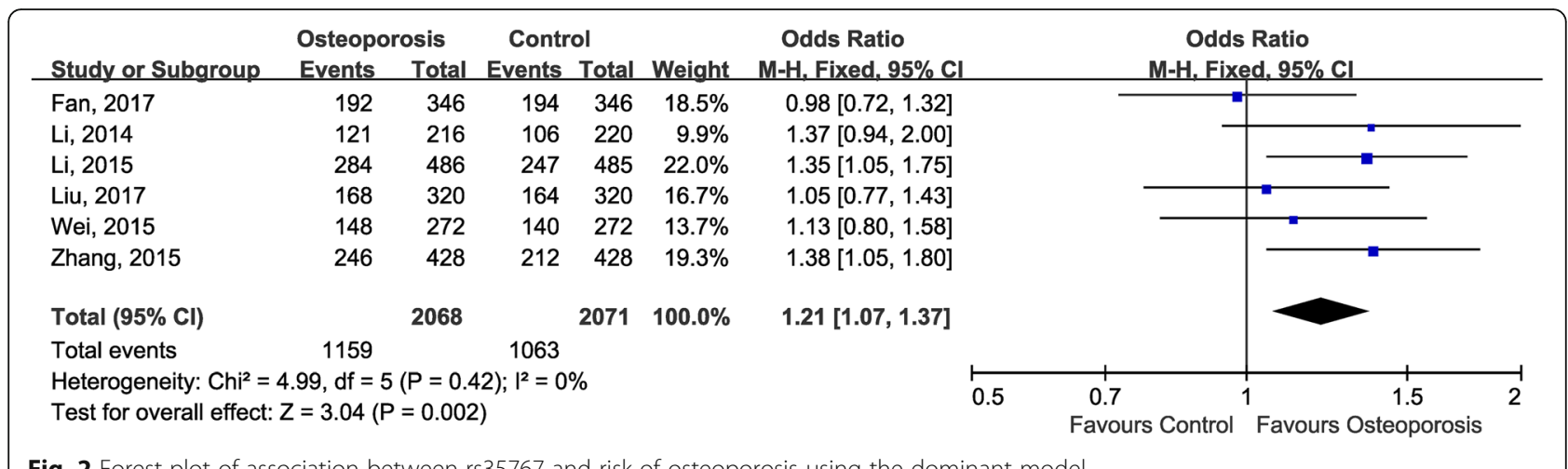

Fig. 2 Forest plot of association between rs35767 and risk of osteoporosis using the dominant model

correlated with bone mass and could serve as independent predictors for the occurrence of osteoporosis and fracture [36].

There have been a series of studies looking into the association between the rs35767 polymorphism in IGF-1 gene and osteoporosis predisposition, while these results acquired were conflicting and as yet no robust evidence was available on this association. With limited sample size of individual study, it is quite difficult to draw a convincing conclusion because of low statistical validity. To overcome this drawback, the systematic review and meta-analysis was a capable alternative [37]. The present study focused on the elderly Chinese population. In the summarized analysis of 2068 osteoporosis cases and 2071 healthy controls, rs35767 polymorphism was investigated to be significantly correlated to osteoporosis susceptibility. To be specific, the $\mathrm{TT}+\mathrm{TC}$ genotype appeared to increase the risk of osteoporosis. However, in the aspect of subgroup-analysis based on gender stratification, only the postmenopausal individuals carrying the TT + TC genotype had a significantly higher osteoporosis vulnerability, which sustained the supposition that postmenopausal women were at a higher risk for osteoporosis.

It should be pointed out that Chen et al. [18] recently published a paper that shared a similar result with our present one. But our study has several methodological advantages. Firstly, we established a more comprehensive literature search strategy for potentially eligible literature, and the literature search and screen yielded six studies including 2068 cases and 2071 controls, while Chen et al. [18] only obtained four studies with 1402 cases and 1405 controls. Except for four studies [17, 26, 28, 29] included by Chen et al., we included two additional recently

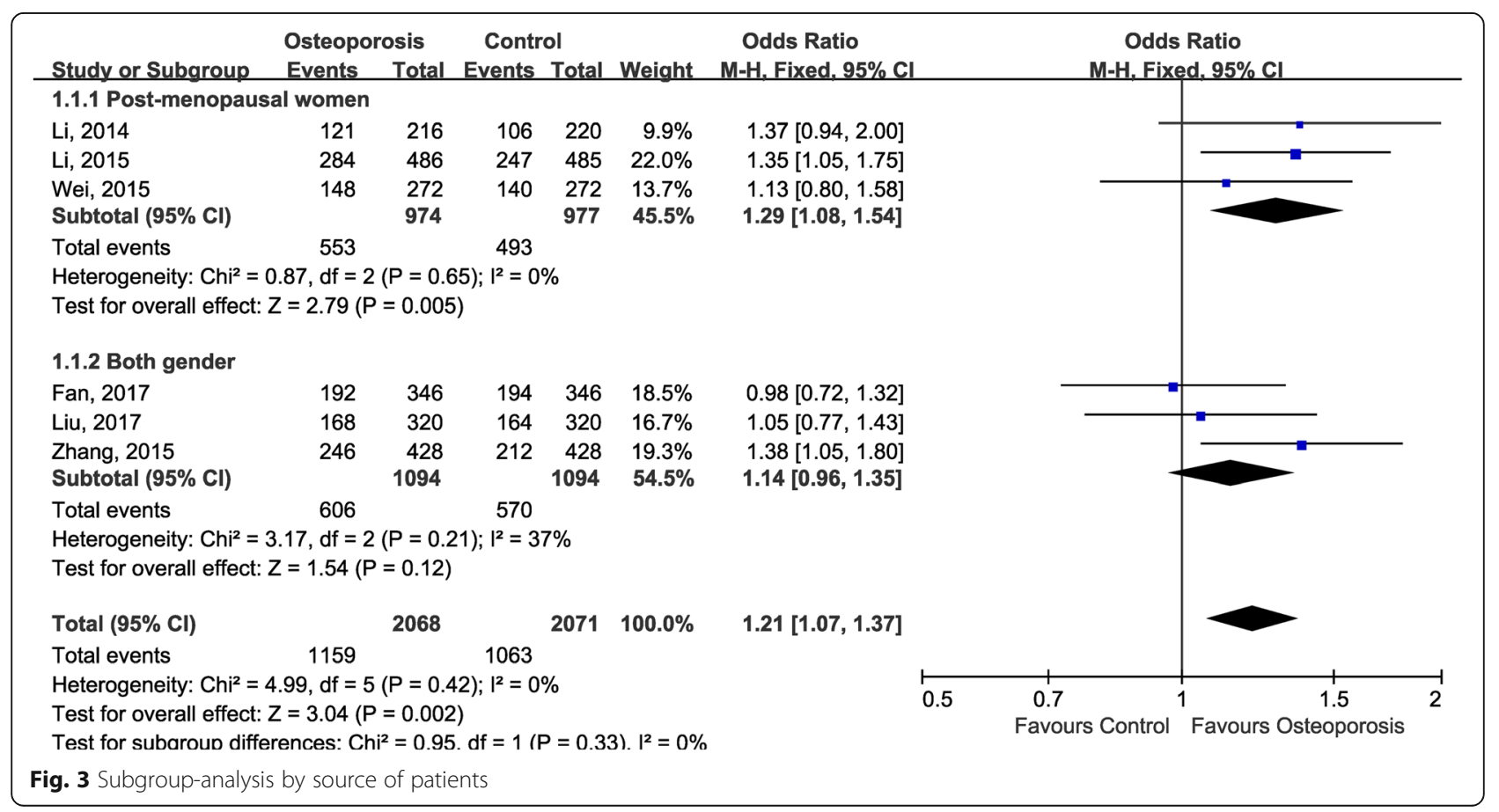




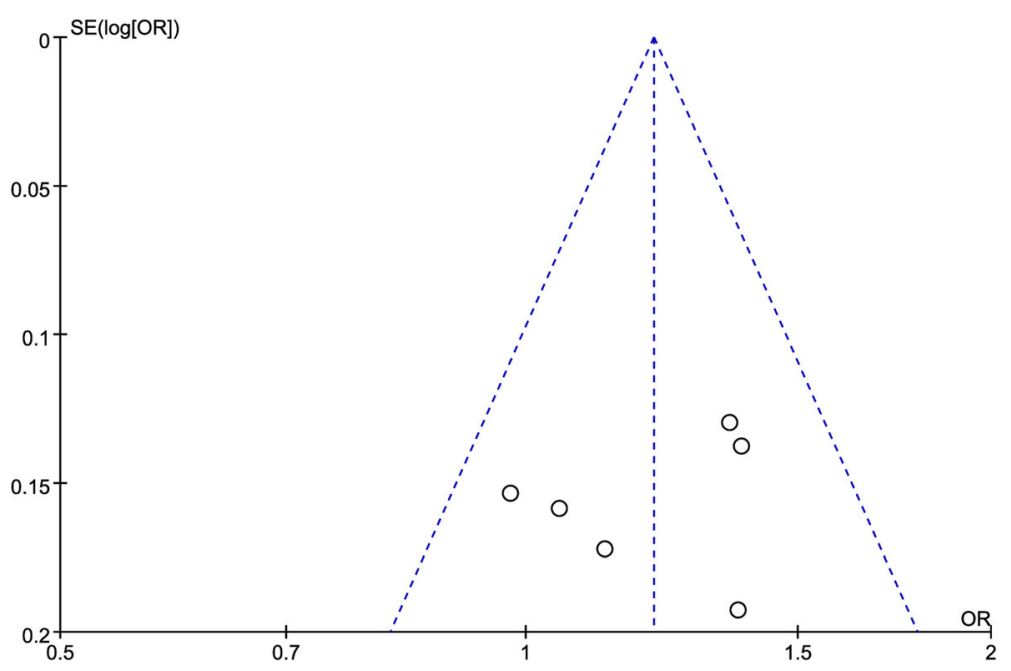

Fig. 4 Funnel plot of association between rs35767 and risk of osteoporosis

published studies $[25,27]$ to increase the statistical power of our study. Secondly, Chen et al. [18] performed their meta-analysis using multiple genetic models of inheritance without applying a correction for multiple testing. Falsepositive results may arise through a failure to correct for multiple testing. In contrast, we employed a model-free approach to avoid this issue. Thirdly, our meta-analysis appraised the HWE of control participants of each included study which also contributed to the credibility of our results.

Although the present meta-analysis has provided a more comprehensive evaluation and precise evidence of the currently available data on the association between the IGF-1 gene polymorphism and osteoporosis, there are still several limitations that should be pointed out. First of all, all the included studies were implemented in Chinese population with limited sample sizes, the result could not be generalized into the whole population. In consideration of the possible ethnic differences, deeper investigations on various populations are warranted to validate the current result. Besides, we only investigated the role of individual locus polymorphism in IGF-1gene separately, but more than one of the loci might interplay with each other and function together to advance osteoporosis. In addition, as with other complex disorders, osteoporosis risk was modulated by multiple genetic factors with synergetic effect other than IGF-1 gene. Therefore, an investigation of the combined interaction of these related genes is necessary to completely elucidate the pathogenesis of osteoporosis. Lastly, only articles in English and Chinese from five databases were retrieved for our meta-analysis, potential relevant articles published in other languages might have been skipped, which might incur inclusion criteria bias [38].

\section{Conclusion}

The findings of our current study suggested a significant association between rs35767 polymorphism and risk of osteoporosis in Chinese postmenopausal women. To confirm the definite gene-disease association between IGF-1 polymorphisms and susceptibility of osteoporosis, additional studies within other ethnic backgrounds are strongly encouraged.

\section{Additional files}

Additional file 1: Figure S1. Forest plot of sensitivity-analysis, given named study is omitted. The sensitivity analysis was performed by sequential excluding each of the eligible studies. While the corresponding pooled odds ratio (OR) appeared to be not significantly affected, indicating a robust and stable result.(TIF $1695 \mathrm{~kb}$ ).

\section{Abbreviations}

BMD: Bone mineral density; CNKI: China National Knowledge Infrastructure; HWE: Hardy-Weinberg equilibrium; IGF-1: Insulin-like growth factor-1; IGFBP: IGF-binding protein.; MeSH: Medical Subject Headings;

NOS: Newcastle-Ottawa Scale; OR: Odds ratio; 95\% Cl, 95\% confidence interval; PRISMA: Preferred Reporting Items for Systematic Review and Meta-Analyses; SNP: Single nucleotide polymorphism

\section{Funding}

This work was not supported by any fund.

Availability of data and materials

The datasets supporting the conclusions of this article are included within the article and in the Additional file 1.

\section{Authors' contributions}

WS produced the idea to this study and he was responsible for making the final version of this paper. SG and ZL did the literature search, screened the potentially eligible studies and evaluated the data from each included study, they both contributed equally to this work. CZ and CM critically revised this manuscript, including important intellectual contents and the grammatical mistakes existed in our original study. All authors read and approved the final manuscript. 


\section{Ethics approval and consent to participate}

Not applicable as this is a meta-analysis of previously published papers.

\section{Competing interests}

The authors declare that they have no competing interests.

\section{Publisher's Note}

Springer Nature remains neutral with regard to jurisdictional claims in published maps and institutional affiliations.

\section{Author details}

'Department of Spine Surgery, The First Affiliated Hospital of Xinjiang Medical University, Xinjiang 830054, China. ${ }^{2}$ Department of Orthopedics, Tongji Hospital, Tongji Medical College, Huazhong University of Science and Technology, Wuhan 430030, Hubei, China. ${ }^{3}$ Department of Orthopedics, General Hospital of the Yangtze River Shipping, Wuhan 430010, China.

\section{Received: 23 December 2017 Accepted: 26 April 2018}

Published online: 10 May 2018

\section{References}

1. Cummings SR, Melton LJ. Epidemiology and outcomes of osteoporotic fractures. Lancet. 2002;359(9319):1761-7.

2. Lorenc R, Gluszko P, Franek E, Jablonski M, Jaworski M, Kalinka-Warzocha E, Karczmarewicz E, Kostka T, Ksiezopolska-Orlowska K, Marcinowska-

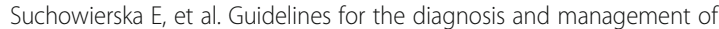
osteoporosis in Poland: update 2017. Endokrynol Pol. 2017;68(5):604-9.

3. Eisman JA. Genetics of osteoporosis. Endocr Rev. 1999;20(6):788-804.

4. Urano T, Inoue S. Genetics of osteoporosis. Biochem Biophys Res Commun. 2014;452(2):287-93.

5. Zhai G, Andrew T, Kato BS, Blake GM, Spector TD. Genetic and environmental determinants on bone loss in postmenopausal Caucasian women: a 14-year longitudinal twin study. Osteoporos Int. 2009;20(6):949-53.

6. Makovey J, Nguyen TV, Naganathan V, Wark JD, Sambrook PN. Genetic effects on bone loss in peri- and postmenopausal women: a longitudinal twin study. J Bone Miner Res. 2007;22(11):1773-80.

7. Kurt O, Yilmaz-Aydogan H, Uyar M, Isbir T, Seyhan MF, Can A. Evaluation of ERalpha and VDR gene polymorphisms in relation to bone mineral density in Turkish postmenopausal women. Mol Biol Rep. 2012;39(6):6723-30.

8. Wu J, Shang DP, Yang S, Fu DP, Ling HY, Hou SS, Lu JM. Association between the vitamin $D$ receptor gene polymorphism and osteoporosis. Biomed rep. 2016:5(2):233-6.

9. Shoukry A, Shalaby SM, Etewa RL, Ahmed HS, Abdelrahman HM. Association of estrogen receptor beta and estrogen-related receptor alpha gene polymorphisms with bone mineral density in postmenopausal women. Mol Cell Biochem. 2015;405(1-2):23-31.

10. Wang H, Gong C, Liu X, et al. J Bone Miner Metab. 2017. https://doi-org.ezpprod1.hul.harvard.edu/10.1007/s00774-017-0862-3.

11. Wang Q, Chen Z, Huang Y, Li Q, Zhu L, Cai X, He G, Xie Y, Liu Q. The relationship between osteoprotegerin gene polymorphisms and bone mineral density in Chinese postmenopausal women. Int Immunopharmacol. 2013;17(2):404-7.

12. Wang F, Cao Y, Li F, Shan J, Wen T. Association analysis between g.18873C >T and g.27522G>a genetic polymorphisms of OPG and bone mineral density in Chinese postmenopausal women. Biomed Res Int. 2014;2014:320828.

13. Giustina A, Mazziotti G, Canalis E. Growth hormone, insulin-like growth factors, and the skeleton. Endocr Rev. 2008;29(5):535-59.

14. Garnero P, Sornay-Rendu E, Delmas PD. Low serum IGF-1 and occurrence of osteoporotic fractures in postmenopausal women. Lancet (London, England). 2000;355(9207):898-9.

15. Kim JG, Roh KR, Lee JY. The relationship among serum insulin-like growth factor-I, insulin-like growth factor-I gene polymorphism, and bone mineral density in postmenopausal women in Korea. Am J Obstet Gynecol. 2002:186(3):345-50

16. Rivadeneira F, Houwing-Duistermaat JJ, Vaessen N, Vergeer-Drop JM, Hofman A, Pols HA, Van Duijn CM, Uitterlinden AG. Association between an insulin-like growth factor I gene promoter polymorphism and bone mineral density in the elderly: the Rotterdam study. J Clin Endocrinol Metab. 2003;88(8):3878-84.
17. Li YK, Wang H, Zhu XW, Guo L, Zuo JL. The polymorphism of insulin-like growth factor-I (IGF-I) is related to osteoporosis and bone mineral density in postmenopausal population. Pakistan j med sci. 2014;30(1):131-5.

18. Chen YC, Zhang L, Li EN, Ding LX, Zhang GA, Hou Y, Yuan W. Association of the insulin-like growth factor-1 single nucleotide polymorphisms rs35767, rs2288377, and rs5742612 with osteoporosis risk: a meta-analysis. Medicine. 2017;96(51):e9231.

19. Moher D, Liberati A, Tetzlaff J, Altman DG, Group P. Preferred reporting items for systematic reviews and meta-analyses: the PRISMA statement. PLoS Med. 2009;6(7):e1000097.

20. GA Wells, B Shea, D O'Connell, J Peterson, V Welch, M Losos, P Tugwell: The Newcastle-Ottawa scale (NOS) for assessing the quality of nonrandomised studies in meta-analyses. Available: http://www.ohri.ca/programs/clinical_ epidemiology/oxford.asp.

21. Thakkinstian A, McElduff P, D'Este C, Duffy D, Attia J. A method for metaanalysis of molecular association studies. Stat Med. 2005;24(9):1291-306.

22. Der Simonian R, Laird N. Meta-analysis in clinical trials. Control Clin Trials. 1986;7(3):177-88.

23. Mantel N, Haenszel W. Statistical aspects of the analysis of data from retrospective studies of disease. J Natl Cancer Inst. 1959;22(4):719-48.

24. Egger M, Davey Smith G, Schneider M, Minder C. Bias in meta-analysis detected by a simple, graphical test. BMJ. 1997:315(7109):629-34.

25. Fan YK, Zhang SY, Liang F, Zhou YY. Association of insulin-like growth factor I gene polymorphisms with the risk of osteoporosis in a Chinese population. Int J Clin Exp Pathol. 2017;10(8):8443-51.

26. Li F, Xing WH, Yang $X J$, Jiang HY, Xia $H$. Influence of polymorphisms in insulin-like growth factor-1 on the risk of osteoporosis in a Chinese postmenopausal female population. Int J Clin Exp Pathol. 2015;8(5):5727-32.

27. Liu XF, Ma FQ, Zhong YB, Wang J, Li JJ, Shan AJ, Yang C. Association of insulin-like growth factor I gene and vitamin $D$ receptor gene polymorphisms with the risk of osteoporosis in a Chinese population. Biomed Res Ind. 2017;28(16):7081-7

28. Wei YK, Ma HL, Guo YZ, Yang BH, Pang WX. Association of the IGF-1 rs35767 and rs972936 polymorphisms with the risk of osteoporosis in a Chinese postmenopausal female population. Gen mol res GMR. 2015;14(4):14325-30.

29. Zhang W, Zhang LC, Chen H, Tang PF, Zhang LH. Association between polymorphisms in insulin-like growth factor-1 and risk of osteoporosis. Gen mol res GMR. 2015;14(3):7655-60.

30. Stewart TL, Ralston SH. Role of genetic factors in the pathogenesis of osteoporosis. J Endocrinol. 2000;166(2):235-45.

31. Ohlsson C, Mohan S, Sjogren K, Tivesten A, Isgaard J, Isaksson O, Jansson JO, Svensson J. The role of liver-derived insulin-like growth factor-I. Endocr Rev. 2009;30(5):494-535.

32. Lee DO, Jee BC, Ku SY, Suh CS, Kim SH, Choi YM, Moon SY, Kim JG. Relationships between the insulin-like growth factor I (IGF-I) receptor gene G3174A polymorphism, serum IGF-I levels, and bone mineral density in postmenopausal Korean women. J Bone Miner Metab. 2008;26(1):42-6.

33. Mohan S. Insulin-like growth factor binding proteins in bone cell regulation. Growth Regul. 1993;3(1):67-70.

34. Matkovic V, Jelic T, Wardlaw GM, Ilich JZ, Goel PK, Wright JK, Andon MB, Smith KT, Heaney RP. Timing of peak bone mass in Caucasian females and its implication for the prevention of osteoporosis. Inference from a crosssectional model. J Clin Invest. 1994;93(2):799-808.

35. Liu JM, Zhao HY, Ning G, Chen Y, Zhang LZ, Sun LH, Zhao YJ, Xu MY, Chen JL. IGF-1 as an early marker for low bone mass or osteoporosis in premenopausal and postmenopausal women. J Bone Miner Metab. 2008;26(2):159-64.

36. Yamaguchi T, Kanatani M, Yamauchi M, Kaji H, Sugishita T, Baylink DJ, Mohan S, Chihara K, Sugimoto T. Serum levels of insulin-like growth factor (IGF); IGF-binding proteins-3, -4 , and -5 ; and their relationships to bone mineral density and the risk of vertebral fractures in postmenopausal women. Calcif Tissue Int. 2006:78(1):18-24.

37. Attia J, Thakkinstian A, D'Este C. Meta-analyses of molecular association studies: methodologic lessons for genetic epidemiology. J Clin Epidemiol. 2003;56(4):297-303.

38. Egger M, Smith GD. Bias in location and selection of studies. BMJ (Clinical research ed). 1998:316(7124):61-6. 